\title{
Malaria specific mortality in lowlands and highlands of Muheza district, north-eastern Tanzania
}

\author{
M.L. KAMUGISHA ${ }^{*}$, S. GESASE ${ }^{1}$, T.D. MLWILO ${ }^{1}$, B.P. MMBANDO ${ }^{1}$, M.D. SEGEJA ${ }^{1}$, D.T. MINJA ${ }^{1}$, J.J. \\ MASSAGA $^{2}$, H.A. MSANGENI ${ }^{1}$, D.R. ISHENGOMA ${ }^{1}$ and M.M. LEMNGE ${ }^{1}$ \\ ${ }^{1}$ National Institute for Medical Research, Tanga Medical Research Centre, P.O Box 5004, Tanga, Tanzania \\ ${ }^{2}$ Centre for Enhancement of Effective Malaria Interventions, P.O Box 9653, Dar es salaam, Tanzania
}

\begin{abstract}
Vital registration of causes of death in Tanzania is incomplete and many deaths occur outside health care settings. Verbal autopsies (VA) are used to determine the underlying cause of death, and the probable diagnosis helps to estimate reasonably cause-specific mortality. In this paper, we report findings of a verbal autopsy survey which involved eight villages in both low and highlands of Muheza district, north-eastern Tanzania. The survey was conducted following a rapid census, which was done to identify households that had lost one or more members within a period of two years from the date of census. Trained research assistants administered VA questionnaires to parents/close relatives. Two physicians reviewed each report independently and a third opinion was sought where there was discordant report between the two. A total of 9,872 households were surveyed and 134 deaths were recorded. A total of 96 (71.6\%) deaths were from lowland villages representing high malaria transmission. Majority (72.4\%) of the reported deaths occurred at home whilst $32.1 \%$ occurred at heath facility settings. Overall, severe malaria was the leading cause accounting for 34.3\% of all deaths. Infants were most affected and accounted for $43.5 \%$ of the total deaths. Pulmonary tuberculosis ranked second (8.2\%) cause of deaths and was exclusively confined to individuals $\geq 15$ years. Probable cause of death could not be determined in $13.4 \%$ of deaths. In conclusion, majority of deaths in rural north-eastern Tanzania occur at home and the immediate causes are usually unknown or not documented. These findings indicate that the verbal autopsy is a useful tool for detecting leading causes of death at community level in the absence of health facility-based data.
\end{abstract}

Key words: verbal autopsy, malaria, mortality, lowland, highland, Tanzania

\section{Introduction}

Worldwide, one out of three deaths is due to an infectious or communicable disease, majority of which occur in developing countries (Gajalakshmi \& Peto, 2004). Globally, information on the cause of death has been useful in supporting the development of evidence-based decision making and in guiding priority setting intervention in public health (Ruzicka et al., 1990). This means, proper estimation of mortality is one of the pre-requisite in understanding the actual picture or determining the magnitude of disease burden (Sankaranarayanan et al., 1996) at the community level especially in rural poor settings. Many deaths in developing countries occur outside health care settings (Lopez, 1990) as a consequence of limited access to health services. Estimation of cause of death becomes more difficult in developing countries because neither health-facility-based information system nor vital registration provides adequate data on the cause of mortality (Gajalakshmi \& Peto, 2006).

Despite high mortality due to infectious diseases in developing countries, the cause of such deaths often goes unrecorded due lack of complete vital registration systems as establishment and maintenance of such systems are always difficult and expensive (Gonghuan et al., 2005; Mathers et al., 2005). According to the World Health Organization, lack of these data hinders the whole process of assessing the global situation on disease specific mortalities as well as monitoring their trends in the most affected countries (WHO, 2004).

In sub-Saharan Africa malaria is the leading cause of morbidity and mortality, especially in children under five years and pregnant women. There are estimated to be 300-500 million clinical cases of malaria each year, about $80 \%$ in tropical Africa (Snow et al., 2005). Coupled with malaria, the situation is worsened further by the upsurge of HIV/AIDS and related opportunistic infections, especially in the adult population (Korenromp et al., 2005; Suri et al., 2006). For various reasons including local beliefs, poor access to health facilities and self-medication practices, many deaths in Africa occur outside the health care systems (Lopez, 1990; de Savigny et al., 2004; Rafael et al., 2006). Most of the information on mortality estimate therefore, emanates from data collected from health facilities which is always an underestimate.

In many of the developing countries verbal autopsy may be a surrogate for death certificates in ascertaining causes of death (Gajalakshmi \& Peto, 2006). Such information may be used to complement the health-facility-based data. Verbal autopsy is a systematic retrospective inquiry of the family members about the circumstances, events, symptoms, and signs of illness prior to death to help determine the underlying cause of death and to classify the broad patterns of mortality.

Malaria is the leading cause of morbidity and mortality in Tanzania. Health facility data indicates that about $19 \%$ of all hospital deaths are due to malaria 
(Kitua, 2003). It is estimated that $11.9 \%$ of deaths occurring at home in Tanzania never consulted health facility for help in the course of their illness (de Savigny et al., 2004). As a result of poor registration systems of vital events, it is often difficult to ascertain reliably the rates and causes of deaths prevailing in developing countries (Gonghuan et al., 2005; Mathers et al., 2005).

In Muheza district, Tanzania, malaria is a leading cause of hospital attendances, admissions, and deaths, and is holoendemic in the lowlands with a prevalence of over 75\% (Lyimo et al., 1991; Ellman et al., 1998). In the highland, on the other hand there is a modest varied level of malaria transmission whereby in some cases a prevalence as low as 33\% has been reported (Ellman et al., 1998). In both zones, malaria transmission is highest during and following the long rainy season, which usually extends from March through July. The average numbers of infective bites per person per year i.e. mean annual entomological inoculation rates; have previously been shown to be about 405 and 34 in the lowlands and highlands, respectively (Ellman et al., 1998). Point prevalence of malaria cases as estimated from community surveys range from 7.4-9.9 in high malaria transmission lowlands, and 2.4-7.4 in the highlands (Ellman et al., 1998).

The aim of this study was therefore, to establish malaria specific mortality at different endemicity in Muheza District, north-eastern Tanzania. This was part of a larger study aimed at establishing an epidemiological map of malaria in north-eastern Tanzania.

\section{Materials and Methods}

\section{Study area and population}

This study was carried out in Muheza District, northeastern Tanzania. The district covers an area of about $4,992 \mathrm{~km}^{2}$ and an estimated human population of 279,473 (URT, 2002). The district is topographically divided into highland and lowland zones. The district is divided into six divisions, 35 wards 175 villages, and 44,124 households. The district is served by 54 dispensaries, four health centres, and one district hospital. Most dispensaries have a catchment population of between 4,000 and 5,000. The major ethnic groups in the district include the Wasambaa (in the highlands) and Wabondei (in the lowlands). The majority of the inhabitants are small-scale farmers of maize, cassava, oranges, coconut, rice and banana.

\section{Study design}

The verbal autopsy survey was carried out in both the highland and lowland villages. Kizerui, Shambangeda, IBC Msasa and Kwelumbizi were selected from the highland zone and were considered to be moderately endemic for malaria. In the lowlands, village selected were Moa, Mwandusi, Jirihini and Kwangena. Selection of the study villages was based on predefined criteria such as proximity to health facility and absence of other malaria intervention activities.

Community meetings were conducted in the selected villages during which the aim and study procedures were explained and discussed with the local population. During these meetings community willingness to participate in the study was also sought. Prior to the verbal autopsy surveys, a quick population census was conducted in all selected villages to establish the sampling unit for the malariometric indices and also for tracing out households with deaths within 2 years from the date of the census. Specially designed population census questionnaires were used to collect the information. The entries included the date of census, name of head of household, tribe, and names of household members, sex, and age, level of education, occupation and history of death in the household within the previous two year from the date of the census. All households with the history of death within the specified 2 year period from the census date were identified.

\section{Data collection}

The WHO standard verbal autopsy questionnaires (WHO, 1999) was adopted and utilised with minor modifications. The modified questionnaires were translated into Kiswahili and back-translated into English to ensure that there was no loss of meaning of information collected as a result of the translation. Three questionnaires were developed which included tools for collecting information on neonatal deaths, post-natal childhood deaths and adults and maternal deaths. The tools contained sections which included background information on the deceased individual and household, and history of illness before death, duration of symptoms and health care seeking including treatment received before death. A team of four field assistants with experience in conducting interview were trained for a period of seven days on how to carry out a verbal autopsy survey. The tools were pre-tested in a pilot survey before they were used in the actual field survey. 
Trained research assistants visited the household of the bereaved families to administer a verbal autopsy questionnaire to parents/close relative that were around at the time of the illness that led to death of their beloved ones. Two physicians reviewed each report independently and a third opinion was sought where there was discordant report between the two.

\section{Results}

A total of 9,872 households were surveyed and 134 deaths were recorded. However, probable cause of death could not be determined in $13.4 \%$ of deaths reported. Significantly more deaths $71.6 \%$ were recorded in the lowland villages (representing high malaria transmission) than in the highland villages. Majority (72.4\%) of the reported deaths occurred at home, whilst $27.6 \%$ occurred at heath care facilities. However there was no variation in proportions of deaths, which occurred at home between lowland and highland areas $(P=0.5)$. Moreover, the proportion of death by gender in both strata was not significantly different $(P=0.3)$ (Table 1).

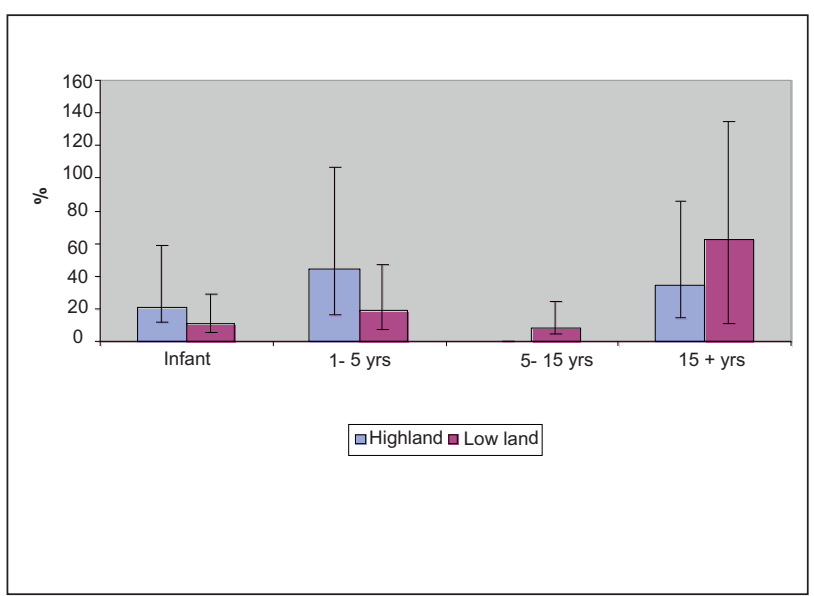

Figure 1: Proportion of deaths by age group with 95\% level in highlands and lowlands

A slightly high non-significant proportion of $31.6 \%$ of deaths occurred at health facility in areas with low malaria transmission as compared to $26.0 \%$ of deaths, which occurred in high transmission areas $(P=0.5)$. Of all deaths that occurred at home, $85.2 \%(69 / 81)$ were in individuals $\geq 5$ years old as compared to $52.8 \%$ $(28 / 53)$ deaths in $<5$ years old $(P=0.001)$.

Table 1: Proportion of deaths by sex, age group and place of death in the highland and lowland villages in Muheza District

\begin{tabular}{|c|c|c|c|}
\hline Variables & Proportion of deaths & & \\
\hline & Highland (n=38) & Lowland $(n=96)$ & P-value \\
\hline \multicolumn{4}{|l|}{$\overline{\text { Sex }}$} \\
\hline Male & $57.9(22)$ & $49.0(47)$ & $P=0.3$ \\
\hline Female & $42.1(16)$ & $51.0(49)$ & \\
\hline \multicolumn{4}{|l|}{ Age groups } \\
\hline$<5$ years & $65.8(25)$ & $29.2(28)$ & $P=0.001$ \\
\hline$\geq 5$ years & $34.2(13)$ & $70.8(68)$ & \\
\hline \multicolumn{4}{|l|}{ Place of death } \\
\hline Health facility & $31.6(12)$ & $26.0(25)$ & $P=0.5$ \\
\hline Home & $86.4(26)$ & $74.0(71)$ & \\
\hline
\end{tabular}

A total of fifty-three (39.6\%) deaths were recorded in children under five years old. A significantly high proportion of deaths that occurred in highland areas were observed among children under the age of five years $(65.8 \%)$ as compared to lowland areas where children under this age category accounted for only $29.2 \%$ of all deaths $(P=0.001)$ (Table 1$)$.
Deaths were distributed unevenly among the age groups (Figure 1). The $1-5$ years and $\geq 15$ years age groups were mostly affected. Whereas the largest proportion of deaths among $\geq 15$ years was observed among those living in the lowlands, most deaths among the 1-5 years olds were from the highland villages. 
Table 2: The percentage (number) of identified causes of death

\begin{tabular}{|c|c|c|c|}
\hline \multirow[b]{2}{*}{ Causes of Death } & \multicolumn{3}{|c|}{ Proportion of death per Strata } \\
\hline & $\begin{array}{l}\text { Highland } \\
(\mathrm{N}=38)\end{array}$ & $\begin{array}{l}\text { Lowland } \\
\qquad(\mathrm{N}=96)\end{array}$ & Total deaths $(\mathrm{N}=134)$ \\
\hline Communicable diseases/conditions & 。 & - & - \\
\hline Severe malaria & $36.8(14)$ & $33.33(32)$ & $34.33(46)$ \\
\hline Pulmonary Tuberculosis & $2.63(1)$ & $10.41(10)$ & $8.21(11)$ \\
\hline Severe anaemia with malaria & $7.9(3)$ & $3.13(3)$ & $4.48(6)$ \\
\hline HIVIAIDS & $2.63(1)$ & $4.17(4)$ & $3.73(5)$ \\
\hline Severe anaemia & $2.63(1)$ & $3.13(3)$ & $2.99(4)$ \\
\hline Meningitis & 01 & $3.13(3)$ & $2.24(3)$ \\
\hline Neonatal septicaemia & $2.63(1)$ & $2.08(2)$ & $2.24(3)$ \\
\hline Severe pneumonia & $2.63(1)$ & $1.04(1)$ & $1.49(2)$ \\
\hline Malaria and severe anaemia in pregnancy & $2.63(1)$ & $\circ$ & $0.75(1)$ \\
\hline Severe malaria and pneumonia & & $1.04(1)$ & $0.75(1)$ \\
\hline Non - communicable diseases/conditions & 。 & 00 & 0 \\
\hline Congestive cardiac failure & $5.26(2)$ & $7.29(7)$ & $6.72(9)$ \\
\hline Renal failure & $2.63(1)$ & $6.25(6)$ & $5.22(7)$ \\
\hline Cancer & 01 & $3.13(3)$ & $2.24(3)$ \\
\hline Diabetes mellitus & $\circ$ & $3.13(3)$ & $2.24(3)$ \\
\hline Severe prematurity & $5.26(2)$ & $1.04(1)$ & $2.24(3)$ \\
\hline Stroke & $2.63(1)$ & 1.04(1) & $1.49(2)$ \\
\hline Embolism & $2.63(1)$ & $\circ 1$ & $0.75(1)$ \\
\hline Animal bite (crocodile) & $2.63(1)$ & $1.04(1)$ & $1.49(2)$ \\
\hline Drowning & 01 & $1.04(1)$ & $0.75(1)$ \\
\hline Birth trauma & $\circ$ & 1.04(1) & $0.75(1)$ \\
\hline Motor traffic accidents & 。 & $1.04(1)$ & $0.75(1)$ \\
\hline Spinal injury & 。 & $1.04(1)$ & $0.75(1)$ \\
\hline Cause was not determined & $18.42(7)$ & $11.46(11)$ & $13.43(18)$ \\
\hline
\end{tabular}

Overall communicable diseases accounted for $70.7 \%$ of all deaths (Table 2). Severe malaria was the leading cause of deaths accounting for $34.3 \%$ of the total deaths. Infants were the most affected age group accounting for $43.5 \%$ of all deaths due to severe malaria. Moreover, 92.9\% (13) of all deaths in $<15$ years old children that occurred in highland villages were due to severe malaria $(P=0.02)$. Pulmonary tuberculosis ranked second $(8.2 \%)$ and was exclusively in the $\geq 15$ years old age group. The proportion of deaths due to pulmonary tuberculosis was significantly higher in the lowlands than highlands $(P<0.05)$. Congestive cardiac failure and renal failure were the most frequent causes of deaths among the non-communicable diseases.

\section{Discussion}

In this study, verbal autopsy method was able to identify leading causes of death among rural communities of north-eastern Tanzania. Similarly, in a recent study in China, verbal autopsy technique was found to perform reasonably well in identifying several leading causes of adult death (Yang et al., 2006). However, in the study in China, the sensitivity of the technique was found to be less satisfactory in detecting deaths from causes of some important diseases. Data from a multi-centre validation study carried out in Tanzania, Ethiopia and Ghana have shown that sensitivity and specificificy of verbal autopsy differ between the three study sites depending on the distribution of the causes of death (Chandramohan et al., 2001).

Our findings have shown that deaths occurring at home are a common phenomenon in rural communities in northern eastern Tanzania. The fact that so many deaths in adults individuals occurred at home suggests for variation in health seeking among different age groups. It is possible that most of the deaths in the underfives were due to delay in health seeking. The reasons for such a trend are many, but may include lack of community knowledge on signs and symptoms of malaria which may provoke health care seeking outside the formal channel which might result into delay in case detection and prompt treatment. Other reasons included poverty, preponderance for traditional healers, lack or poor health care services, long distances to the nearest health facility as well as cost sharing scheme. In many cases, the use of self medication using wrong or counterfeit drugs sold by drug vendors and in kiosks is also likely to exacerbate the delay in health seeking behaviour (Alilio et al., 1997).

The observed differences in mortality due to severe malaria among children below five years between the two strata might be accounted for by the 
low immunity in individuals living in areas with low to moderate transmission in the highland. There is consistent evidence that shows that immunity against malaria results from cumulative exposure to malaria parasite leading to acquired immunity at younger age for residents of high transmission intensity. The observed pattern could be explained by extended risk for severe Plasmodium falciparum infection toward older age for residents of low transmission due to delay in attaining immunity against severe malaria. The relatively high proportion of death due to tuberculosis is of great concern. The prevalence of tuberculosis is high, especially during this era of HIV/AIDS (Ministry of Health, unpubl.). Similar observations have been reported by AMMP (1997).

From the VA survey, it is clear that early health seeking behaviour in these communities must be emphasized. This should always focus to the most vulnerable groups, the underfives and pregnant women. It is crucial for the health authorities in the area to make sure that frequent appropriate health educations aimed at changing individual health seeking behaviour is promoted. Training of mothers on detection of dangers signs and management of malaria is likely to reduce the mortality among the children (Frank et al 2006; Makundi et al., 2006). Emphasis should also be given to women attending antenatal clinics for the importance of seeking medical attentions for their children and this should go hand in hand with giving proper education to traditional birth attendants.

In order to have proper surveillance, and generate accurate data on malaria disease burden and related morbidity and mortality in areas with limited access to health care facility, VA surveys is likely to act as an important complement in portraying the accurate picture of the case specific mortality provided that those involved are well trained and have all the necessary data collection tools and availability of physicians to verify the probable cause of deaths for the reported mortalities.

The fact that non-communicable accounted for about a third of the total causes of death indicates an increase in the burden of such conditions, which have received little attention by health authorities. There is therefore need for urgent measures to intervene on this growing public health problem.

In conclusion, the majority of deaths among rural communities in Tanzania still occur at home and usually the immediate cause is usually unknown. This study has shown that verbal autopsy technique may be utilized to bridge the gap in the establishment of probable cause of deaths that occur outside health care facilities.

\section{Acknowledgements}

We would like to thank all village leaders and members of the communities in the surveyed villages for their cooperation during the study. We are grateful to families of the bereaved for their readiness to provide information that was required in the survey. Special thanks are to Margaret Hamis, Japheth Kimbesa and Dominic Shauri for their assistance during the population census and verbal autopsy surveys. This study received financial support from the Ministry of Health and Social Welfare, Tanzania.

\section{References}

Alilio, M.S., Kamugisha, M.L., Msuya, F., Massaga, .J.J., Salum, F.M.Q, \& Njunwa, K.J (1997) Availability and Utilization of antimalarial drugs at community level in Same District, North Eastern Tanzania. Malaria and Infectious Diseases in Africa 6, 8-12.

Chandramohan, D., Setel, P. \& Quigley, M. (2001) Effect of misclassification of causes of death in verbal autopsy: can it be adjusted? International Journal of Epidemiology 30, 509-514.

de Savigny, D., Mayombana, C., Mwageni, E., Masanja, H., Minhaj, A., Mkilindi, Y., Mbuya, C., Kasale, H. \& Reid, G. (2004) Care seeking patterns for fatal malaria in Tanzania. Malaria Journal 3, 27.

Elllman, R., Maxwell, C., Finch, R. \& Shayo, D. (1998) Malaria and anaemia at different altitudes in the Muheza district of Tanzania: childhood morbidity in relation to level of exposure to infection. Annals of Tropical Medicine and Parasitology 92, 741-753.

Frank, M.R., Bygbjerg, I.C. \& Helle, S. (2006) Early home-based recognition of anaemia via general danger signs, in young children, in a malaria endemic community in north-east Tanzania. Malaria Journal 5, 111.

Gajalakshmi, V. \& Peto, R. (2004) Verbal autopsy of 80000 adult deaths in Tamilnadu, South India. BMC Public Health 4, 471-421.

Gajalakshmi, V. \& Peto, R. (2006) Commentary: verbal autopsy procedure for adult deaths. International Journal of Epidemiology 35, 748750 . 
Gonghuan, Y., Jianping, H., Ke, Q.R., Jeimin, M., Chalapati, R. \& Alan, D.L (2005) Mortality registration and surveillance in China: history, current situation and challenges. Population Health Metrics 3, 1-9.

Kitua, A.Y. (2003) Malaria control in the context of integrated management of childhood illness in Tanzania: the challenges ahed. Tanzania Health Research Bulletin 5, 1-4.

Korenromp, E.P., Williams, B.G., de Vlas, S.J., Gouws, E., Gilks, C.F., Ghys, P.D. \& Nahlen, B.L (2005) Malaria attributable to the HIV-1 epidemic, sub-Saharan Africa. Emerging Infectious Disease 11,1410-1419.

Lopez, A.D (1990) Causes of death: an assessment of global patterns of mortality around 1985. World Health Statistics Quarterly 43, 91-104.

Lyimo, E.O., Msuya, F.H., Rwegoshora, R.T., Nicholson, E.A., Mnzava, A.E., Lines, J.D. \& Curtis, C.F. (1991) Trial of pyrethroid impregnanted bednets in an area of Tanzania holoendemic for malaria. Part 3. Effects on the prevalence of malaria parasitaemia and fever. Acta Tropica 49, 157-163.

Makundi, E.A., Malebo, H.M., Mhame, P., Kitua. A.Y. \& Warsame. M. (2006) Role of traditional healers in the management of severe malaria among children below five years of age: the case of Kilosa and Handeni Districts, Tanzania. Malaria Journal 5, 58.

Mathers, C.D., Mafat, D., Inoue, M., Tao, C. \& Lopez, A.D. (2005) counting the dead and what they died of: an assessment of the global status of cause of death data. Bulletin of the World Health Organization 83, 171-55.
AMMP (1997) The Policy Implications of Adult Morbidity and Mortality Project: end of Phase I Report. Ministry of Health Dar es Salaam, United Republic of Tanzania.

Rafael, M.E., Taylor, T., Magill, A., Lim, Y., Girosi, F. \& Allan, R. (2006) Reducing the burden of childhood malaria in Africa: the role of improved diagnostics. Nature. Accessed at http:// www.nature.com/diagnostics. December 19, 2006.

Ruzicka, L.T. \& Lopez, A.D (1990) The use of causeof death statistics for health situation assessment: national and international experience. World Health Statics Quarterly 43, 249-258.

Sankaranarayanan, R., Saminathan, R. \& Black, R.J. (1996) Global variations in cancer survival. Study Group on Cancer Survival in Developing Countries. Cancer 78, 2461-2464.

Snow, R.W., Guerra, C.A., Noor, A.M., Myint, H.Y. \& Hay, S.I. (2005) The global distribution of clinical episoded of Plasmodium falciparum. Nature 434, 214-217.

Suri, V., Bhalla, A., Sharma, N., Jain, S. \& Varma, S. (2006) HIV immunosupression and malaria: is there a correlation? Indian Journal of Medical Sciences 60, 376-379.

URT (2002) Household Budget Survey 2000/01. National Bureau of Statistics, p.21. Dar es Salaam, United Republic of Tanzania.

WHO (1999) A standard Verbal Autopsy Method for Investigating Causes of Death in Infants and Children. WHO/CDS/CSR/IRS/99/4.

Yang, G., Rao, C., Ma, J., Wang, L., Wan, X., Dubrovsky, G. \& Lopez, A.D. (2006) Validation of verbal autopsy procedures for adult deaths in China. International Journal of Epidemiology 35, 741-748. 